\title{
Rohr, Christian, Extreme Naturereignisse im Ostalpenraum : Naturerfahrung im Spätmittelalter und am Beginn der Neuzeit
}

\section{François Walter}

\section{OpenEdition}

Édition électronique

URL : http://journals.openedition.org/ifha/1611

DOI : 10.4000/ifha.1611

ISSN : 2198-8943

\section{Éditeur}

IFRA - Institut franco-allemand (sciences historiques et sociales)

\section{Référence électronique}

François Walter, «Rohr, Christian, Extreme Naturereignisse im Ostalpenraum : Naturerfahrung im Spätmittelalter und am Beginn der Neuzeit », Revue de l'IFHA [En ligne], Date de recension, mis en ligne le 01 janvier 2008, consulté le 22 septembre 2020. URL : http://journals.openedition.org/ifha/1611 ; DOI : https://doi.org/10.4000/ifha.1611

Ce document a été généré automatiquement le 22 septembre 2020.

(CIFHA 


\title{
Rohr, Christian, Extreme
}

Naturereignisse im Ostalpenraum : Naturerfahrung im Spätmittelalter und am Beginn der Neuzeit

\author{
François Walter
}

1 Ce gros livre est le résultat d'une thèse d'habilitation à l'Université de Salzbourg où enseigne actuellement son auteur. Partant du constat qu'il existe une abondante littérature décrivant des épisodes calamiteux mais qu'il s'agit le plus souvent d'études de cas dispersés chronologiquement et géographiquement, C.R. a l'ambition de la synthèse. Il a choisi les Alpes orientales en incluant sous cette appellation l'actuelle Autriche, une partie de la Bavière, la Slovénie et même une partie de l'Italie du Nord. La période retenue va de 1200 à 1600 , chevauchant donc la traditionnelle coupure entre le Moyen Âge et le début des temps modernes. Ce point de vue s'est avéré particulièrement judicieux car la recherche met bien en évidence les changements qui interviennent dès le XVe s. et se confirment avec la Réforme. C.R. adopte résolument une approche d'histoire culturelle pour essayer de définir des cultures de la catastrophe, différenciées selon les périodes et les lieux. Les événements l'intéressent bien sûr aussi, mais l'essentiel se ramène à la perception, au sens et à la gestion des catastrophes naturelles, l'hypothèse étant que le contexte interprétatif doit privilégier la lecture religieuse des phénomènes et mobiliser autant qu'il se peut l'expérience des générations passées. L'objectif est de modéliser les paramètres de la perception des catastrophes naturelles.

2 Les premiers chapitres conservent le caractère de l'écrit académique avec leurs fastidieuses considérations historiographiques. Des premiers catalogues de tremblements de terre au XVe s. jusqu'aux recherches pluridisciplinaires actuelles, l'auteur ne nous fait grâce d'aucune référence sans oublier les révérences obligées aux auteurs les plus en vue institutionnellement. En revanche, la présentation des sources est sans aucun doute nécessaire : annales et chroniques mais aussi un certain nombre 
de documents administratifs (les comptes où sont signalés les montants investis dans les réparations après dommages), les prédications et les sources iconographiques en forment l'essentiel. Un cahier de documents en couleur complète avantageusement l'ouvrage.

D'entrée C.R. rappelle opportunément que l'interprétation providentialiste n'exclut pas forcément une pensée attentive à comprendre le fonctionnement des mécanismes naturels. Cela est évident pour les inondations. Ce l'est moins pour des phénomènes perçus comme inexplicables, telle l'arrivée soudaine d'une nuée d'insectes ou les pluies de sang (en réalité des précipitations véhiculant des poussières et des sables colorés). Sept chapitres très inégaux en taille présentent chacun un type de catastrophe naturelle : successivement tremblements de terre, glissements de terrains, inondations, avalanches, autres événements météorologiques extrêmes (grêle, tempêtes et autres sécheresses ou grands froids), insectes et ravageurs, phénomènes célestes associés à des calamités (comètes, éclipses et pluies de sang). Les différents épisodes sont bien sûr décrits dans leur déroulement et leurs effets, ainsi que par la manière dont les communautés concernées réagissent et maîtrisent les conséquences destructives. On trouvera utilement en annexe la liste des catastrophes recensées dans l'espace des Alpes orientales. Mais l'essentiel est dans la lecture qui en est proposée. Ainsi, au Moyen Âge, la plupart des phénomènes naturels ne donnent pas forcément prise à une lecture providentialiste. On évite même de les rapporter systématiquement à un cadre interprétatif théologique ou eschatologique. La chose est particulièrement évidente pour les inondations (le plus gros chapitre du livre avec plus de 200 pages !). Les matrices bibliques des récits de calamités ne sont pas mobilisées, probablement parce que la récurrence des hautes eaux, a fortiori dans des espaces vallonnés, où la masse liquide ne peut pas s'étaler comme elle le fait dans les régions planes des basses terres, ne semble aucunement évoquer un quelconque déluge universel. Les choses changent au XVIe s. quand les références à l'avertissement divin, voire à l'action punitive ou plus généralement rétributive de Dieu envahissent le discours. Ici, C.R. souligne l'impact de plusieurs séries d'éléments : d'abord, la perception concrète des effets du refroidissement climatique (le début du Petit Âge glaciaire) ; ensuite, les incertitudes eschatologiques très liées aux temps de la Réforme protestante ; enfin, le recours à des modèles rhétoriques de la tradition antique, mis en valeur par l'humanisme et amenés d'Italie au XVe s. Ainsi le mot diluvium devient une référence pour évaluer l'importance d'une inondation. Par exemple, Enea Piccolomini (le futur pape Pie II) l'utilise dans son récit d'une expérience vécue en Autriche en 1445. Les lettres du même mot, prises comme chiffres romains et additionnées, donnent la date de 1567, année de fortes inondations. La Renaissance valorise aussi l'astrologie, ce qui fait qu'on associe plus volontiers autour de 1500 le passage des comètes ou les éclipses à des présages d'événements catastrophiques. Outre une impressionnante moisson d'informations nouvelles sur des régions alpines encore peu étudiées, cette mise en évidence d'un changement interprétatif au début des Temps modernes est sans aucun doute le principal apport de cette recherche qui fait de son auteur l'un des meilleurs spécialistes de l'histoire des calamités tardimédiévales. On objectera simplement à cette thèse, mais cela reste à démontrer, qu'un changement qualitatif des sources utilisées au moment de la rupture épistémologique pourrait biaiser les résultats. Si cela était, les interprétations providentialistes auraient, sans que les sources en témoignent, toute leur pertinence dès avant le XVes. 
4 François Walter (Université de Genève) 